\title{
Estudos hidrodinâmicos do escoamento em caixa de gordura empregada no tratamento preliminar dos efluentes de cozinha industrial
}

\author{
Hydrodynamic flow studies in the grease trap employed \\ in the primary treatment of effluents from industrial kitchen
Waina Bella de Castro Junqueira', Claudio Milton Montenegro Campos², Ronaldo Fia ${ }^{3}$, Fátima Resende Luiz Fia ${ }^{4}$, Fabiana Amorim ${ }^{5}$

\begin{abstract}
口
\end{abstract}
\section{RESUMO}

Efluentes de cozinhas industriais se caracterizam pela geração de elevado teor de óleos e gorduras. Esses produtos notadamente causam sérios problemas ambientais, sendo obrigatória a instalação de unidades de pré-tratamento no local onde são gerados, e com isso surge a importância das caixas de gordura (CGS), destinadas a esse fim. O desempenho das CGs tem relação direta com as condições processuais e hidrodinâmicas que ocorrem em seu interior. O presente estudo teve o objetivo de comparar as características hidrodinâmicas da CG funcionando sob duas condições operacionais distintas: flotação gravitacional e flotação forçada (auxiliada por difusores de ar). Realizaram-se quatro testes hidrodinâmicos na CG, sendo o primeiro e segundo testes com água limpa a uma vazão constante e o terceiro e quarto testes com efluente do Restaurante Universitário (RU), cuja vazão era variável em função das atividades do RU. Aplicaram-se a metodologia de distribuição do tempo de residência(DTR) e a técnica de estímulo e resposta utilizando cloreto de potássio (KCL) como elemento traçador. Os resultados hidrodinâmicos no primeiro teste operacional apontaram características entre o escoamento pistonado e o escoamento dispersivo, com moderada intensidade de dispersão e certa mistura. O segundo teste, com aeração, mostrou tendência ao escoamento em mistura completa com grande intensidade de dispersão. O terceiro teste, por flotação gravitacional, mostrou tendência a fluxo pistonado, enquanto o quarto teste demonstrou fluxo dispersivo, tendendo a um elevado grau de mistura. Observaram-se, em todos os testes, aparentes desvios causados provavelmente por zonas estagnadas (mortas), curtos-circuitos e fenômenos de recirculação, fazendo com que o volume útil da CG não fosse plenamente utilizado. Apesar de não fazer parte do escopo deste trabalho, a eficiência na remoção de óleos e graxas foi maior quando aplicada a flotação gravitacional, cuja mistura tende à pistonada, o que auxilia o grau de aquiescência e permite maior acúmulo de gordura flotada. Quanto à flotação forçada por meio dos aeradores, grande

\begin{abstract}
Effluents from industrial kitchens are characterized by high-generated content of oils and fats. These products cause serious environmental problems, and it is mandatory to install pretreatment units in the places where they are generated. Hence the importance of grease traps, intended for this purpose, whose performance is directly related to the processual and hydrodynamic conditions inside. This study aimed to compare the hydrodynamic characteristics of greaser trap (GT) working under two different operating conditions: gravitational flotation and forced flotation (aided by air diffusers). Four hydrodynamic tests were performed in the GT, the $1^{\text {st }}$ and $2^{\text {nd }}$ tests with clean water at a constant flow rate and the 3rd and 4th tests with wastewater from the university restaurant, with variable flow. The methodology applied to evaluate the residence time distribution (RTD) was the technique of stimulus/response using potassium chloride $(\mathrm{KCl})$ as a tracer element. The hydrodynamic results in the $1^{\text {st }}$ operating test indicated features between slug flow and dispersive flow, with moderate dispersion intensity and some mixing. The $2^{\text {nd }}$ operating test, under aeration, showed tendency of complete mixing with great dispersion intensity. The $3^{\text {rd }}$ test, by gravitational flotation, tended to become plug-flow, while the $4^{\text {th }}$ test was characterized as dispersive flow, tending to a high degree of mixing. In all tests, apparent deviations, probably caused by stagnant areas or dead zones, short-circuits and internal recirculation phenomena, were observed, confirming that the useful volume of GT was not fully utilized. Although not part of the scope of this work, the efficiency in removing oils and greases was higher when the gravitational flotation was applied, whose mixture tends to the plug-flow type, which assists the degree of acquiescence and allows a greater accumulation of floated fat. As for the forced flotation through
\end{abstract}

口-

'Doutora em Recursos Hídricos em Sistemas Agrícolas (Recursos Hídricos e Saneamento Ambiental) pela Universidade Federal de Lavras (UFLA) - Lavras (MG), Brasil. Arquiteta da Pró-Reitoria de Infraestrutura da Universidade Federal de Juiz de Fora (UFJF) - Juiz de Fora (MG), Brasil.

${ }^{2}$ Doutor em Environmental Engineering pela University of Newcastle Upon Tyne - United Kingdom. Professor Titular da UFLA - Lavras (MG), Brasil.

${ }^{3}$ Doutor em Engenharia Agrícola (Recursos Hídricos e Ambientais) pela Universidade Federal de Viçosa (UFV) - Viçosa (MG), Brasil. Professor Adjunto do Departamento de Engenharia e Coordenador do Curso de Graduação em Engenharia Ambiental e Sanitária da UFLA - Lavras (MG), Brasil.

${ }^{4}$ Doutora em Engenharia Agrícola pela UFV - Viçosa (MG), Brasil. Professora Adjunta do Departamento de Engenharia da UFLA - Lavras (MG), Brasil.

${ }^{5}$ Doutoranda na UFLA - Lavras (MG), Brasil.

Endereço para correspondência: Waina Bella de Castro Junqueira - Rua Barão do Cotegipe, 339, Apto. 01 - Centro - Leopoldina (MG), Brasil - E-mail: waina.junqueira@yahoo.com.br Recebido em: 06/05/14 - Aceito em: 15/08/16 - Reg. ABES: 134506 
parte da gordura é oxidada e, portanto, minimizada, formando uma escuma de cor clara (branca) e de baixíssima espessura na parte superior da CG. Todavia, por se tratar de elevado grau de mistura, a eficiência de retenção se mostrou, como previsto, inferior ao processo gravitacional.

Palavras-chave: óleos e gordura; caixa de gordura; hidrodinâmica; modelos matemáticos. the aerators, much of the fat is oxidized and thus minimized, forming a light color slag (white) and of very low thickness at the top of the CG. However, because of its high degree of mixture, the retention efficiency, as expected, was shown to be lower than the gravitational process.

Keywords: oils and fat; fat trapper; hydrodynamics; mathematical models.

\section{INTRODUÇÃO}

Efluentes provenientes de cozinhas industriais, em sua maioria, não são tratados ou não recebem tratamento adequado. Tais resíduos caracterizam-se por elevadas vazões intermitentes e com grande teor de óleos e gorduras. Quando lançados diretamente na natureza, causam problemas ambientais tanto ao solo como aos corpos hídricos receptores, pois os óleos e as gorduras possuem baixa solubilidade e alta resistência à degradação, tornando-se um dos constituintes orgânicos mais complexos ao tratamento. Associados a outros compostos intermediários gerados durante sua biodegradação, provocam interferências contraproducentes nas estações de tratamento de esgoto (ETEs), tais como toxicidade, efeitos inibitórios aos micro-organismos, varredura (wash-out) do lodo, trazendo diminuição da biomassa ativa e, consequentemente, obrigando a diminuição da carga orgânica biológica aplicada (ORSSATO; HERMES; VILAS BOAS, 2010; YANG; CHEN; CHEN, 2012).

Os óleos e as gorduras, identificados analiticamente por meio do parâmetro de qualidade intitulado "óleos e graxas" (O\&G), não podem ser facilmente tratados por processos biológicos convencionais, portanto é indicado pré-tratamento no local onde são gerados. As caixas de gordura (CGs), unidades destinadas a esse fim, atuam na retenção superficial de partículas menos densas que o meio para posterior remoção (METCALF \& EDDY, 2003; JORDÃO \& PESSÔA, 2005).

Nesse contexto, nas CGs convencionais, a remoção de O\&G ocorre por processos de flotação gravitacional, porém os processos por flotação forçada (auxiliada por ar difuso) vêm ganhando fundamental importância (PALMEIRA et al., 2009) como alternativa no tratamento das águas residuárias ricas nesses compostos, como as provenientes de cozinhas e restaurantes. Entretanto, o desempenho dessas unidades se relaciona diretamente com as condições hidrodinâmicas predominantes em seu interior, e o conhecimento dessas condições contribui para dimensioná-las e aperfeiçoá-las quando já construídas, pois permite verificar se os critérios adotados no projeto estão realmente adequados, podendo ou não ser legitimados.

Para determinar o comportamento hidrodinâmico, torna-se necessário, como condição básica, o conhecimento da distribuição do tempo de residência (DTR) da infinidade de moléculas constituintes do fluido, as quais podem se dispersar e percorrer diferentes caminhos, despendendo tempos distintos para percorrer o volume líquido, sujeito a vários distúrbios. A DTR é obtida mediante o método de estímulo e resposta, em que o estímulo é a injeção de um elemento traçador no afluente, no tempo zero, com substância inerte ao meio investigado. A resposta consiste nas leituras das concentrações das amostras coletadas no efluente, imediatamente após a injeção do traçador e sequencialmente ao longo do tempo, que deve idealmente corresponder a, pelo menos, três vezes o tempo de residência teórico $\left(\mathrm{TRH}_{\text {teórico }}\right)$ calculado em projeto, o qual representa o quociente entre o volume da unidade e a vazão aplicada (LEVENSPIEL, 2000; CAPELA et al., 2009; LOURENÇO \& CAMPOS, 2009).

A avaliação da curva DTR gerada pela concentração de saída em função do tempo monitorado permite simular ou predeterminar resultados por meio de modelos de escoamento ideal do fluido (pistonado e mistura completa), pois configuram uma ampla faixa, dentro da qual as unidades reais se enquadram (CAPELA et al., 2009; LOURENÇO \& CAMPOS, 2009). Porém, na prática, o escoamento segue modelos que consideram a dispersão das partículas do fluido, ou seja, modelos intermediários entre os dois extremos citados, como os modelos de dispersão e o modelo de células agitadas em série (PAOLI \& VON SPERLING, 2013). Teoricamente, os modelos pistonados, em se tratando de reatores biológicos, apresentam maior eficiência do que aqueles de comportamento hidrodinâmico, tendendo para mistura completa. $\mathrm{Na}$ aplicação de tais modelagens são obtidos os principais parâmetros hidrodinâmicos, como tempo médio de residência hidráulica $\left(\mathrm{TRH}_{\text {médio }}\right)$, correspondente ao tempo de residência médio real observado, e variância $\left(\sigma^{2}\right)$, capazes de estimar o número de dispersão $(d)$ e o número de células agitadas em série $(N)$, favorecendo, assim, a determinação do tipo de mistura e das prováveis anomalias relacionadas ao escoamento nas unidades reais, como, por exemplo: dispersão, recirculação, curtos-circuitos e zonas estagnadas.

O objetivo principal deste estudo foi comparar as características hidrodinâmicas de uma CG funcionando um período por flotação gravitacional e outro por flotação forçada, respectivamente, utilizando-se como fluido, inicialmente, água limpa (primeiro e segundo testes) e, posteriormente, água residuária proveniente do restaurante universitário (RU) (terceiro e quarto testes).

\section{MÉTODOS}

O experimento foi conduzido na CG devidamente projetada como unidade de tratamento preliminar, construída em um espaço anexo ao RU do Campus da Universidade Federal de Lavras (UFLA). A referida unidade possui a seguinte configuração: volume de $5,24 \mathrm{~m}^{3}$; comprimento 
e largura úteis de 3,55 e 0,85 m, respectivamente; profundidade útil variando de 1,57 a 1,90 m, ao longo do comprimento, a fim de permitir o escoamento e a retirada do lodo decantando (Figuras 1 e 2).
A CG trabalhou com duas condições operacionais distintas: primeiramente, flotação gravitacional, com o sistema de aeração desligado; e, posteriormente, flotação forçada, auxiliada por difusores

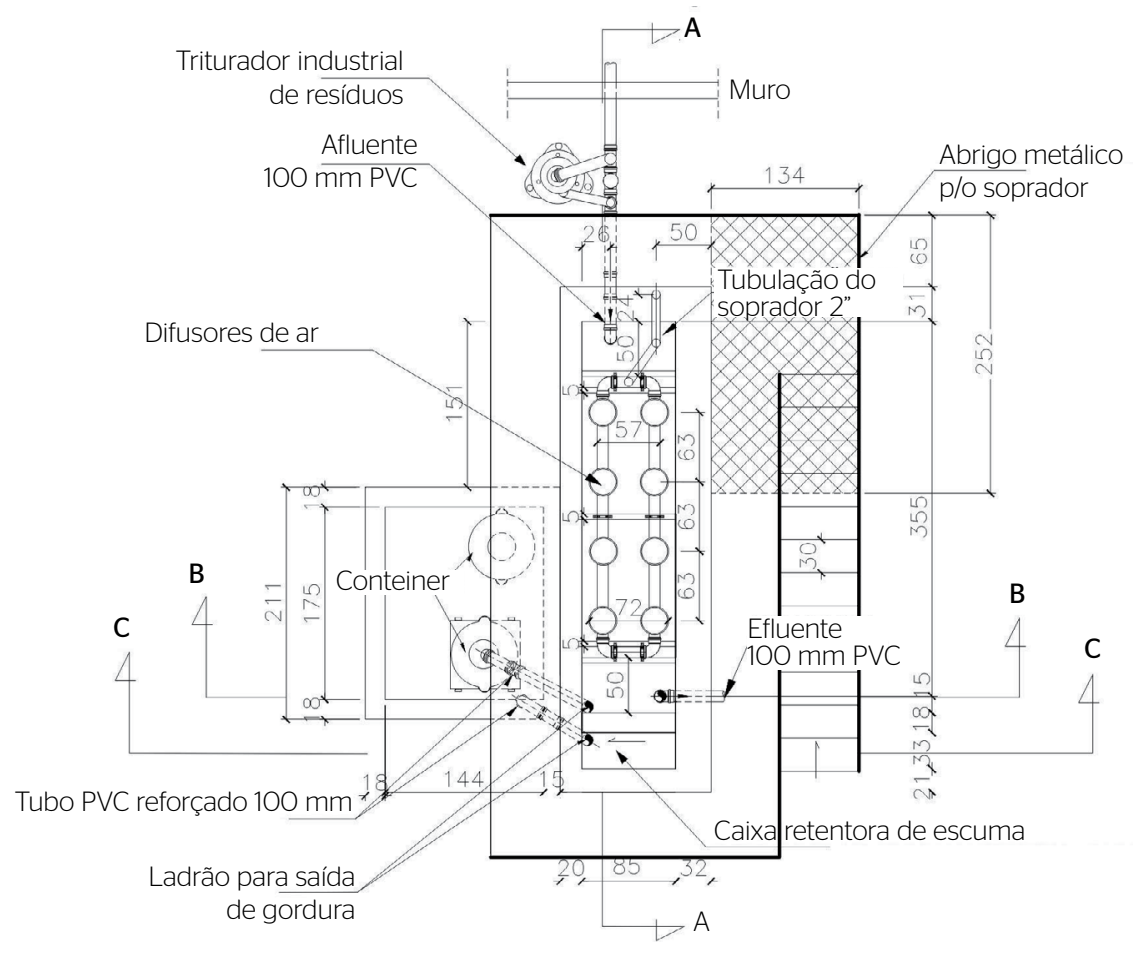

Figura 1 - Planta baixa da caixa de gordura (medidas em $\mathrm{cm}$ ).

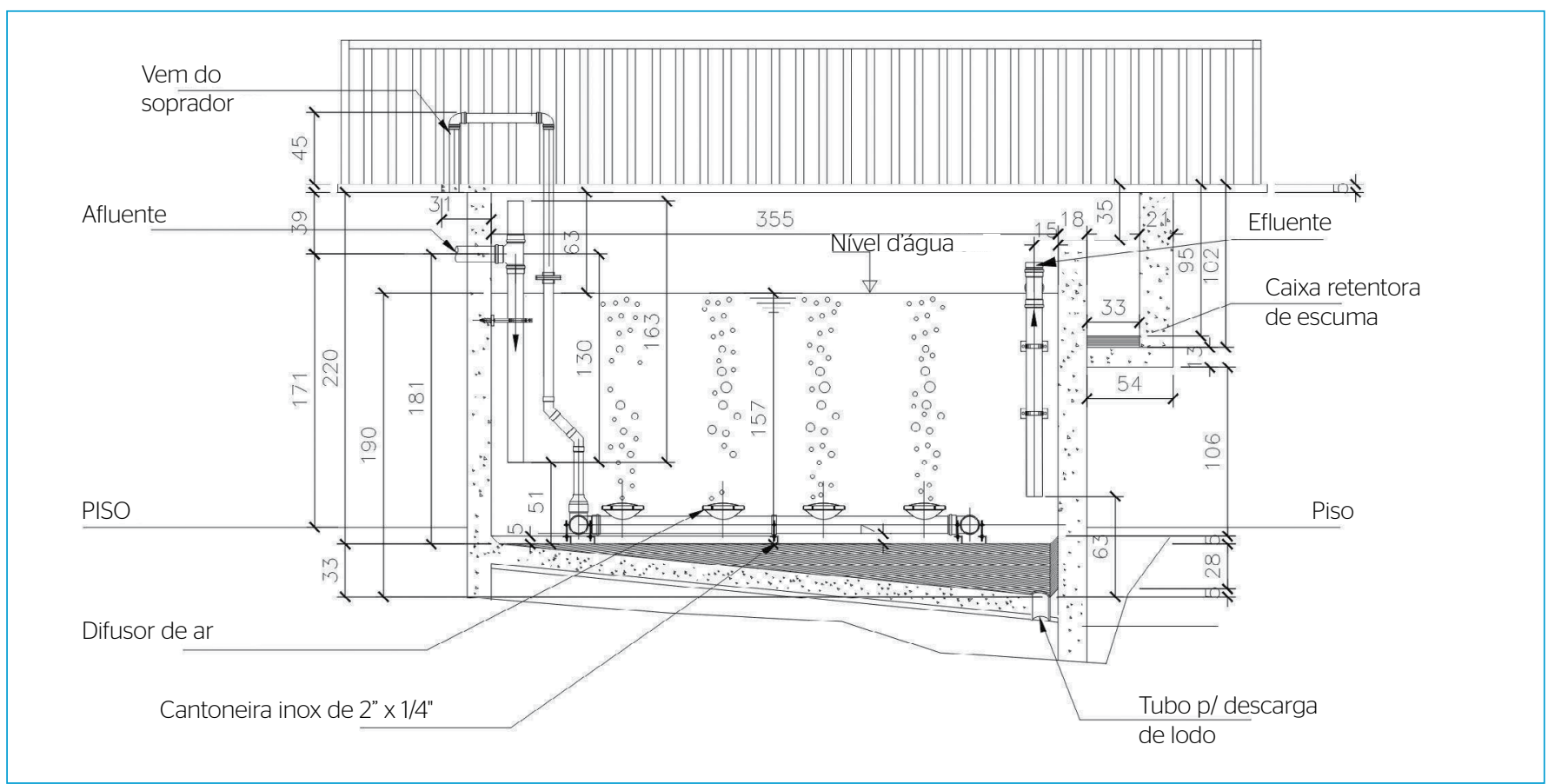

Figura 2 - Corte A-A da caixa de gordura (medidas em $\mathrm{cm}$ ). 
de ar submersos, acionados por soprador com potência de 1,5 kW, 1740 RPM, equivalente a uma densidade de potência $\varphi=0,286 \mathrm{kWm}^{-3}$. $\mathrm{O}$ ar foi aplicado por meio de 8 difusores (com diâmetro de $230 \mathrm{~mm}$, bolha fina) construídos em PVC com membrana EPDM a uma taxa constante de $4,2 \mathrm{~m}_{\text {ar }}^{3} \cdot \mathrm{m}^{-3}$.

\section{Delineamento dos testes hidrodinâmicos}

O primeiro e o segundo testes hidrodinâmicos, respectivamente com a CG sob flotação gravitacional e flotação forçada, foram realizados com água oriunda da rede de abastecimento da UFLA, com vazão praticamente constante. O terceiro e o quarto testes hidrodinâmicos foram conduzidos com água residuária do RU, com grandes oscilações de vazão, contendo resíduos constituídos de gorduras dispersas, floculadas e emulsionadas, restos de alimento provenientes da higienização, do processamento, da preparação e do cozimento.

A metodologia utilizada nos testes foi a de estímulo e resposta segundo a técnica de pulso, citada por Campos (1990) e Levenspiel (2000), empregando-se o cloreto de potássio (KCl) como elemento traçador, cujo preparo seguiu a metodologia descrita por Campos (1990). Inicialmente, antes da introdução do traçador, coletou-se uma amostra do fluido (branco) para verificar se havia $\mathrm{K}^{+}$no meio. Após a injeção do $\mathrm{KCl}$, realizada no tubo de entrada (afluente), as amostras foram coletadas na saída (efluente) da CG, em intervalos de tempo regulares. Para a leitura das concentrações de $\mathrm{K}^{+}$, utilizou-se fotômetro de chama da marca Quimis, modelo Q398M2, 220v-188W, com faixa de medição de leitura de 0-100 ppm, nos testes com água limpa, enquanto nos testes com água residuária do RU foi utilizado fotômetro de chama da marca Micronal, modelo $4 \mathrm{~B}$ $262,220 \mathrm{~V}$, calibrado para a faixa de medição de leitura de 0-20 ppm. As características operacionais de todos os testes hidrodinâmicos encontram-se na Tabela 1.

O $1^{\circ}$ teste ocorreu durante um período de aproximadamente 1.920 minutos, quase 6 vezes maior que o $\mathrm{TRH}_{\text {teórico }}$ (334 minutos), com a CG sendo alimentada de forma ininterrupta (com água limpa). Já no $2^{\circ}$ teste a CG foi alimentada com o mesmo fluido de modo intermitente, simulando o horário de funcionamento do RU, das $7 \mathrm{~h}$ às $13 \mathrm{~h} 30 \mathrm{~min}$ (6h30min por dia). Ao término das coletas, desligava-se o sistema de aeração e interrompia-se a água na entrada da CG. A duração total do teste, considerando-se o horário das $17 \mathrm{~h} 30 \mathrm{~min}$ sem alimentação, foi de 4.710 minutos, aproximadamente 4 vezes maior que $\mathrm{o} \mathrm{TRH}_{\text {teórico }}$ (1.232 minutos).

Os demais testes (terceiro e quarto) com a CG sendo alimentada com efluente do RU ocorreram de modo intermitente. Foram consideradas as $6 \mathrm{~h} 30 \mathrm{~min}$ de funcionamento do RU somadas às $17 \mathrm{~h} 30 \mathrm{~min}$ em que não havia vazão. $\mathrm{O} 3^{\circ}$ teste teve duração de 8.980 minutos, tempo aproximadamente 17 vezes maior que o $\mathrm{TRH}_{\text {teórico }}$ (523 minutos). Já o $4^{\circ}$ teste totalizou 9.030 minutos, tempo aproximadamente 20 vezes maior que o $\mathrm{TRH}_{\text {térico }}$ (462 minutos) do experimento.

Ao final dos testes, foram obtidos os resultados das concentrações de $\mathrm{K}^{+}$versus os intervalos de tempo de coleta (C.t) que permitiram a construção dos gráficos (curvas de DTR) com o software Excel'. Posteriormente, as curvas DTR foram ajustadas ao modelo matemático de dispersão de grande intensidade descrito em Levenspiel (2000). Para a comprovação dos resultados de todos os testes, empregou-se o modelo de células agitadas em série, representando as condições hidráulicas intermediárias entre as unidades de mistura completa $\mathrm{e}$ de escoamento pistonado, que, por hipótese, pode ser assumido pela CG ao funcionar com aeração e sem aeração.

Os parâmetros hidrodinâmicos determinados foram: $\mathrm{TRH}_{\text {teórico' }}$ obtido pela Equação 1; $\mathrm{TRH}_{\text {médio }}$, obtido por meio da metodologia apresentada por Levenspiel (2000) e Metcalf \& Eddy (2003) (Equação 2); número de dispersão $(d)$, considerando-se o modelo de dispersão de grande intensidade (quando d $>0,01$ ) descrito em Levenspiel (2000) (Equação 5); variância da distribuição do tempo médio de residência $\left(\sigma^{2}\right)$, calculada pela (Equação 3); variância normalizada da distribuição $\left(\sigma_{\theta}^{2}\right)$, determinada pela (Equação 4). $\mathrm{O} N$ foi determinado pela (Equação 6). Para obter $d$ e $N$, foi utilizada $\sigma^{2}{ }_{\theta}$, bem como a derivação das equações apresentadas em Levenspiel (2000) (Equação 7) com a função gama de $N, \mathrm{~g}(\mathrm{t})$ (Equação 8), metodologia citada por Kadlec \& Wallace (2008). Nesta última, o parâmetro $N$ foi selecionado para minimizar a soma dos erros quadrados entre a função $g(t)$ e os dados do traçador, sendo fixados os valores de $\mathrm{TRH}_{\text {médio }}$ obtidos por meio dos testes.

Tabela 1 - Características de operação do ensaio hidrodinâmico.

\begin{tabular}{|c|c|c|c|c|}
\hline Descrição & $\begin{array}{l}10 \\
\text { teste }\end{array}$ & $\begin{array}{l}2^{\circ} \\
\text { teste }\end{array}$ & $\begin{array}{c}30 \\
\text { teste }\end{array}$ & $\begin{array}{l}4^{\circ} \\
\text { teste }\end{array}$ \\
\hline Vazão observada (L.min'1) & 15,69 & 15,69 & 36,98 & 41,80 \\
\hline Vazão observada $\left(m^{3} \cdot\right.$ dia $\left.^{-1}\right)$ & 23,00 & 6,12 & 14,42 & 16,30 \\
\hline Traçador utilizado & \multicolumn{4}{|c|}{$(\mathrm{KCl})$} \\
\hline Massa de $\mathrm{KCl}$ & \multicolumn{4}{|c|}{$500 \mathrm{~g}$} \\
\hline Massa de $\mathrm{K}^{+}$adicionada & \multicolumn{4}{|c|}{$262,22 \mathrm{~g}$} \\
\hline Forma de injeção & Pulso ( $(15 \mathrm{~s})$ & Pulso ( $\sim 9 \mathrm{~s})$ & \multicolumn{2}{|c|}{ Pulso ( 3 s) } \\
\hline Volume de injeção de $\mathrm{KCl}$ & \multicolumn{2}{|c|}{$1,5 \mathrm{~L}$} & \multicolumn{2}{|c|}{$2 L^{*}$} \\
\hline Concentração de KCl & \multicolumn{2}{|c|}{$333,33 \mathrm{~g} \cdot \mathrm{L}^{-1}$} & \multicolumn{2}{|c|}{$250,00{\mathrm{~g} . \mathrm{L}^{-1}}^{-1}$} \\
\hline Concentração de $K^{+}$ & \multicolumn{2}{|c|}{$174,82{\mathrm{~g} . \mathrm{L}^{-1}}^{-1}$} & \multicolumn{2}{|c|}{$131,11 \mathrm{~g} \cdot \mathrm{L}^{-1}$} \\
\hline $\begin{array}{l}\text { Intervalo de coleta } \\
\text { (minutos) }\end{array}$ & 30 & 10 & 20 & 10 \\
\hline $\begin{array}{l}\text { Número de coletas } \\
\text { (amostras) }\end{array}$ & 65 & 160 & 139 & 280 \\
\hline
\end{tabular}

*Mudança no volume de injeção por se encontrarem cristais de sal no fundo do recipiente.

$\mathrm{KCl}$ : cloreto de potássio. 


$$
\begin{aligned}
& T R H_{\text {teórico }}=\frac{V}{Q} \\
& T R H_{\text {medio }}=T D H_{\text {real }}=\frac{\int_{0}^{\infty} t \cdot C(t)}{\int_{0}^{\infty} C(t)} \cdot d t \\
& \sigma^{2}=\frac{\int_{0}^{\infty} t^{2} \cdot C(t)}{\int_{0}^{\infty} C(t)} \cdot d t-\left(T R H_{\text {medio }}\right)^{2} \\
& \sigma_{\theta}^{2}=\frac{\sigma^{2}}{\left(T R H_{\text {médio }}\right)^{2}} \\
& \sigma_{o}^{2}=\frac{\sigma^{2}}{\left(T R H_{\text {medio }}\right)^{2}}=2\left(\frac{D}{u \cdot L}\right)-2\left(\frac{D}{u \cdot L}\right)^{2} \cdot\left(1-e^{-D / u . L}\right) \\
& N=\frac{1}{\sigma_{\theta}^{2}}=\frac{\overline{t^{2}}}{\sigma^{2}} \\
& N=\frac{1}{2 d-2 d^{2} \cdot\left(1-e^{-1 / d}\right)} \\
& g(t)=\frac{N}{T R H_{\text {médio }} \Gamma(N)} \cdot \frac{\left(N \cdot T R H_{\text {téricio }}\right)^{N-1}}{T R H_{\text {médio }}} \cdot e^{\left(\frac{-N . T R H \text { térico }}{\text { TRHmétio }}\right)}
\end{aligned}
$$

Em que:

V: volume da unidade de tratamento $\left(\mathrm{L}^{3}\right)$;

$\mathrm{Q}: \operatorname{vazão}\left(\mathrm{L}^{3} \mathrm{~T}^{-1}\right)$;

C: concentração de saída do traçador $\left(\mathrm{ML}^{-3}\right)$;

t: tempo decorrido com a análise com traçador $(\mathrm{T})$;

$\mathrm{D}$ : coeficiente de dispersão que caracteriza o grau de mistura $\left(\mathrm{L}^{2} \mathrm{~T}^{-1}\right)$;

$\mathrm{u}$ : velocidade média de escoamento $\left(\mathrm{L} \mathrm{T}^{-1}\right)$;

L: comprimento do percurso longitudinal na unidade (L);

$d=\mathrm{D} / \mathrm{uL}$ : número de dispersão (adimensional);

$\Gamma(N)$ : função gama de $N$, para números inteiros [fatorial de $\mathrm{N}^{-1}\left(\mathrm{~d}^{-1}\right)$ ].

Para a comprovação dos parâmetros hidrodinâmicos encontrados, foram utilizados cálculos numéricos e programa de integração por Simpson desenvolvido por Campos (1990), empregado na simulação dos resultados. O programa computacional foi feito na linguagem Visual Basic'. Ao mesmo tempo, seguindo a metodologia proposta por Metcalf \& Eddy (2003), estimaram-se alguns índices que avaliam a eficiência volumétrica, a extensão do escoamento, a dispersão, os curtos-circuitos e as zonas estagnadas (mortas). Esses índices corroboram a avaliação mais cuidadosa do desempenho hidráulico da CG.

Para a avaliação dos resultados do número de dispersão, consideraram-se: dispersão desprezível, indicando escoamento pistonado, para $d$ tendendo a zero, e grande dispersão, indicando escoamento em mistura completa, para $d$ tendendo ao infinito $(\infty)$ (LEVENSPIEL, 2000; METCALF \& EDDY, 2003); condições de escoamento se aproximando do pistonado, para $d$ em torno de 0,2 ou menos, e se aproximando das condições de mistura completa, para $d$ em torno de 3,0 ou mais (VON SPERLING, 1996). Já a avaliação da intensidade de dispersão baseou-se na Tabela 2.
Quanto à avaliação dos resultados de $N$, foram considerados: padrão de escoamento pistonado, para $N$ tendendo ao infinito $(\infty)$, e mistura completa, para $N$ tendendo a 1 . Valores intermediários indicam escoamento disperso (LEVENSPIEL, 2000).

Primeiramente, foram relacionados o $1^{\circ}$ e o $3^{\circ}$ testes hidrodinâmicos, com a CG sob flotação gravitacional, e, posteriormente, o $2^{\circ} \mathrm{e}$ o $4^{\circ}$ testes, com a CG sob flotação forçada.

\section{RESULTADOS E DISCUSSÃO}

Os resultados do $1^{\circ}$ e do $3^{\circ}$ testes hidrodinâmicos, respectivamente com água limpa e com água residuária do RU com o sistema de aeração da CG desligado, são apresentados nas Figuras 3 e 4 e nas Tabelas 3 e 4 . Cabe destacar que o $3^{\circ}$ teste ocorreu após 146 dias do início das atividades na CG.

No $1^{\circ}$ teste, a curva de DTR apresenta aparente simetria, de modo semelhante aos modelos de pequena a moderada intensidade de dispersão (LEVENSPIEL, 2000), confirmando a situação imposta de vazão aplicada constante. As dosagens de $\mathrm{K}^{+}$se apresentam estáveis como em escoamento pistonado, sem grandes picos de saída. A presença de uma pequena cauda indica a existência de zonas estagnadas, e a saída de $\mathrm{K}^{+}$durante a primeira hora de avaliação mostra a ocorrência de

Tabela 2 - Avaliação da intensidade de dispersão.

\begin{tabular}{c|c} 
Dispersão (d) & Intensidade \\
Zero & Nenhuma \\
\hline$<0,05$ & Pequena* $^{*}$ \\
\hline 0,05 a 0,25 & Moderada \\
\hline$>0,25$ & Grande \\
\hline
\end{tabular}

Fonte: Metcalf \& Eddy (2003)

*Para Levenspiel (2000), quando D/uL<0,01.

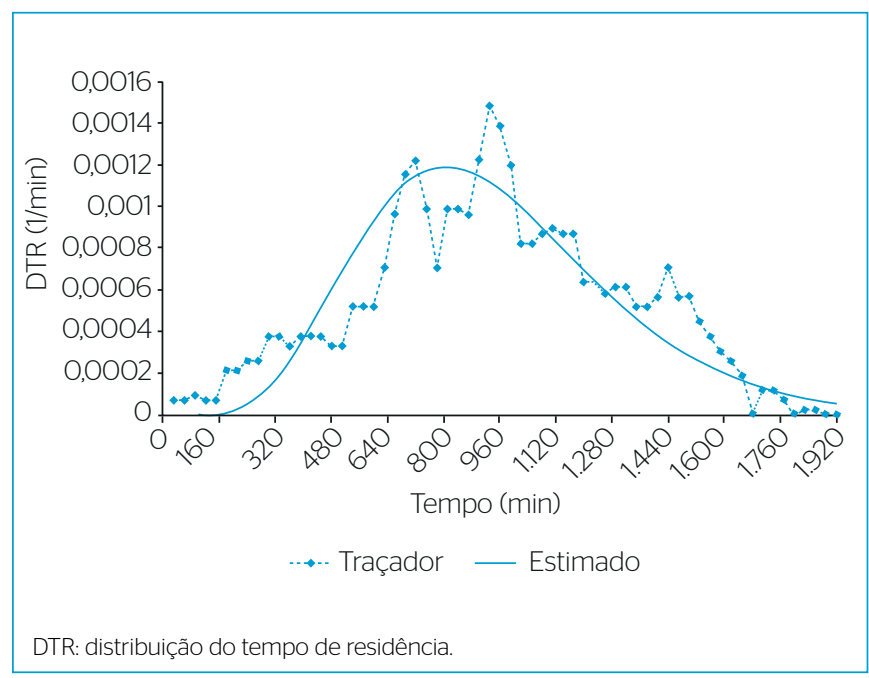

Figura 3 - Curva e ajuste da distribuição do tempo de residência pela função gama no 10 teste, totalizando 32 horas (1.920 minutos). 
caminhos preferenciais ou curtos-circuitos. O teste foi interrompido com 32 horas (1.920 min), haja vista que o final da curva não retornou para valores próximos a 0 . Nota-se retardo na liberação do traçador comprovado pela diferença entre $\mathrm{TRH}_{\text {médio }}$ e $\mathrm{TRH}_{\text {teórico }}$, este superado

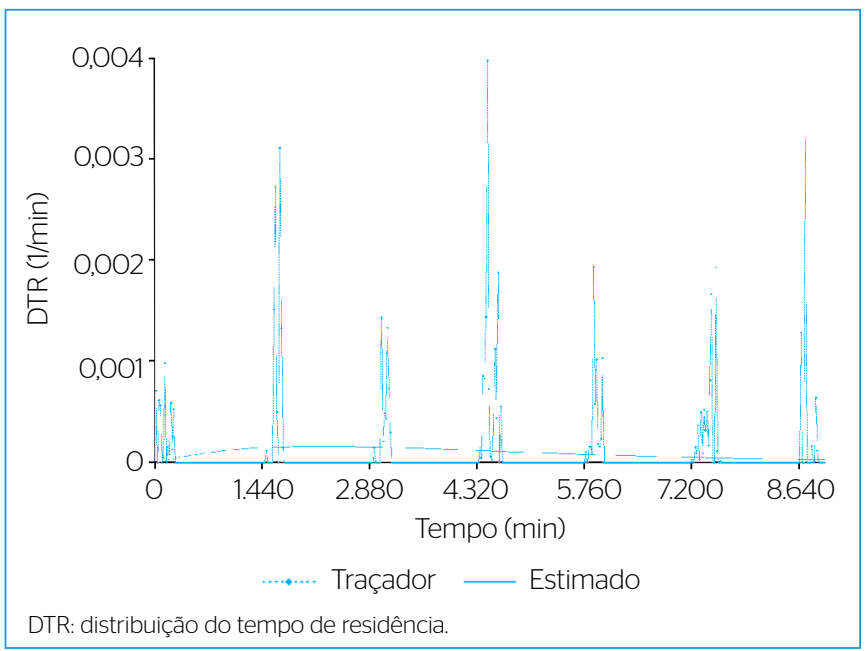

Figura 4 - Curva e ajuste da distribuição do tempo de residência pela função gama no $3^{\circ}$ teste, totalizando 149,67 horas ( 8.980 minutos).

Tabela 3 - Resultados dos parâmetros hidrodinâmicos no $1^{\circ}$ e $3^{\circ}$ testes, com a caixa de gordura funcionando por flotação gravitacional com o sistema de aeração desligado.

\begin{tabular}{l|c|c|c}
\hline Parâmetro & Equação & 10 teste & 30 teste \\
\hline $\mathrm{TRH}_{\text {teórico }}$ & 1 & $334 \mathrm{~min}=0,23$ dias & $523 \mathrm{~min}=0,36$ dias \\
\hline $\mathrm{TRH}_{\text {médio }}$ & 2 & $971 \mathrm{~min}=0,67 \mathrm{dias}$ & $4.475 \mathrm{~min}=3,11$ dias \\
\hline$\sigma^{2}$ & 3 & $129.991,16 \mathrm{~min}^{2}$ & $6.895 .159,15 \mathrm{~min}^{2}$ \\
\hline$\sigma^{2} \theta$ & 4 & 0,14 & 0,34 \\
\hline$d$ & 5 & 0,07 & 0,22 \\
\hline$N$ & $6,7 \mathrm{e} 8$ & 7 & $3 \mathrm{e} \mathrm{2}$ \\
\hline
\end{tabular}

$\mathrm{TRH}_{\text {téricio: }}$ tempo de residência hidráulica teórico; $\mathrm{TRH}_{\text {médio }}$ : tempo de residência hidráulica médio. em aproximadamente três vezes. Ainda, com $d<0,2$ (VON SPERLING, 1996), confirma-se a aproximação às condições de escoamento tipo pistão. Igualmente, o valor de $N$ reforça esse conceito, uma vez que, quanto maior o $N$, menor o grau de mistura (MENDONÇA, 2002). A intensidade de dispersão pode confirmar-se como moderada (para $d$ entre 0,05 e 0,25) (METCALF \& EDDY, 2003).

No $3^{\circ}$ teste, a curva de DTR não apresentou bom ajuste, possivelmente em virtude das dificuldades com o teste real, como: mudanças de vazão, natureza variada do afluente, grande acúmulo de lodo decantado (aproximadamente 1,00 m) e elevado teor de escuma (aproximadamente $0,19 \mathrm{~m}$ ) com a utilização parcial do volume líquido total da CG.

Como explicação para o fato de o $\mathrm{TRH}_{\text {médio }}$ ser superior ao $\mathrm{TRH}_{\text {teórico }}$ é sugerida a ocorrência de retardo na liberação do traçador, confirmando o indício de zonas mortas em função da conformação prismática da CG. Ainda, o valor de $d$ bem próximo a 0,2 (VON SPERLING, 1996) aponta para um escoamento tendendo a pistonado. Já o valor de $N$ entre zero e infinito indica escoamento disperso, em que a dispersão pode ser resultado dos seguintes fatores: escoamento; turbulência dos gases formados no processo anaeróbio que ocorria no interior da CG; inversão térmica causada pela variação da temperatura no interior da CG; e, ainda, condições de alimentação intermitente com variações constantes da vazão, no período das atividades de almoço no RU (6h30min diárias), causando variações da carga hidráulica e orgânica. Uma vez cessado o escoamento, os íons de $\mathrm{K}^{+}$provavelmente se distribuíam internamente em algumas regiões (zonas mortas) e/ou por adsorção ao lodo decantado ou na escuma superficial, devido, principalmente, aos processos advectivos e dispersivos (FERREIRA, 2012; METCALF \& EDDY, 2003).

Os resultados do $2^{\circ}$ e do $4^{\circ}$ testes hidrodinâmicos, com a CG funcionando por flotação forçada (sistema de aeração ligado), respectivamente com água limpa e com água residuária, são apresentados nas Figuras 5 e 6 e nas Tabelas 5 e 6 . Cabe destacar que o $4^{\circ}$ teste teve início oito dias após a partida da CG.

Tabela 4 - Resultados dos índices encontrados na avaliação do desempenho hidráulico da caixa de gordura funcionando por flotação gravitacional no $1^{\circ}$ e $3^{\circ}$ testes hidrodinâmicos.

\begin{tabular}{|c|c|c|c|}
\hline Índices & $1^{\circ}$ teste & 30 teste & Interpretação (METCALF \& EDDY, 2003) \\
\hline $\begin{array}{l}\text { Índice de avaliação da eficiência volumétrica } \\
\left(\mathrm{TDH}_{\text {médio }} / \mathrm{TDH}_{\text {teórico }}\right)\end{array}$ & 2,91 & 8,56 & $\begin{array}{l}\text { Valor diferente de } 1 \text { indica perturbações de escoamento (zonas mortas e/ou } \\
\text { curtos-circuitos) devido à conformação prismática da CG, com cantos e arestas. }\end{array}$ \\
\hline $\begin{array}{l}\text { Índice de curto-circuito } \\
\left(\mathrm{TDH}_{\mathrm{i}} / \mathrm{TDH}_{\text {teórico }}\right)\end{array}$ & 0,18 & 0,04 & $\begin{array}{l}\text { Valor menor que } 1 \text { indica curtos-circuitos e valor próximo a zero indica } \\
\text { alguma mistura. }\end{array}$ \\
\hline $\begin{array}{l}\text { Indice para estimativa do índice de dispersão } \\
\left(\mathrm{IDM}=\mathrm{TDH}_{90 \%} / \mathrm{TDH}_{10 \%}\right)\end{array}$ & 3,13 & 5,44 & $\begin{array}{l}\text { Valor distante do escoamento em mistura completa (IDM=22), aproximando- } \\
\text { se do pistonado (IDM=1) com alguma dispersão. }\end{array}$ \\
\hline
\end{tabular}

$\mathrm{TRH}_{\text {médio }}$ : tempo de residência hidráulica médio; $\mathrm{TRH}_{\text {teórico: }}$ tempo de residência hidráulica teórico; $\mathrm{CG}$ : caixa de gordura; $T R \mathrm{H}_{\text {pico: }}$ : tempo em que a concentração de pico do traçador é registrada na saída; TRH: tempo em que o traçador foi detectado pela primeira vez; $\mathrm{TRH}_{10 \%}, \mathrm{TRH}_{50 \%}$ e $\mathrm{TRH}_{90 \%}$ : tempo em que, respectivamente, 10, 50 e $90 \%$ da massa do traçador passam pela saída da unidade; IDM: índice de dispersão de Morril. 


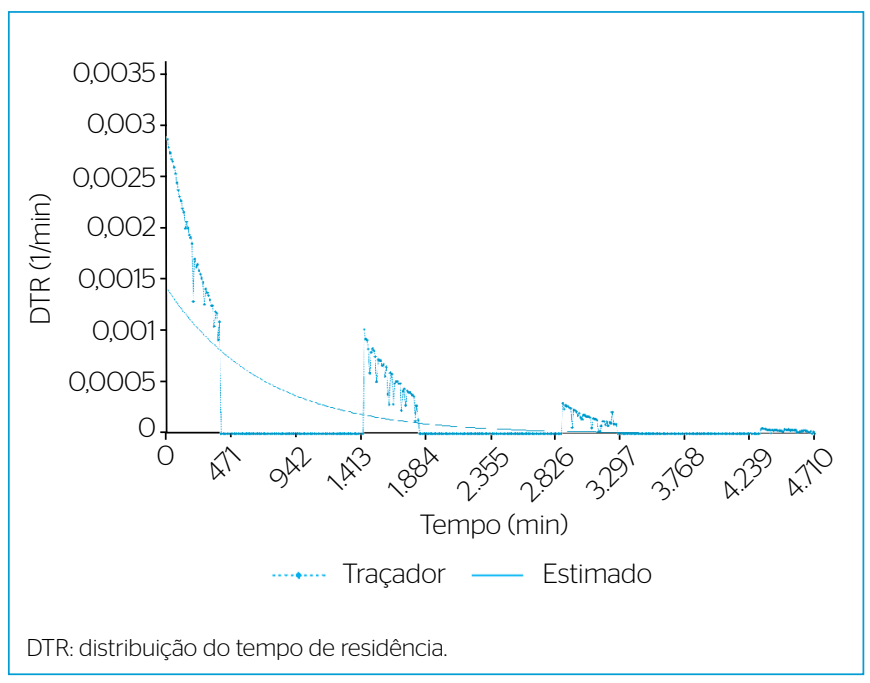

Figura 5 - Curva e ajuste da distribuição do tempo de residência pela função gama no $2^{\circ}$ teste, totalizando 78,5 horas (4.710 minutos).

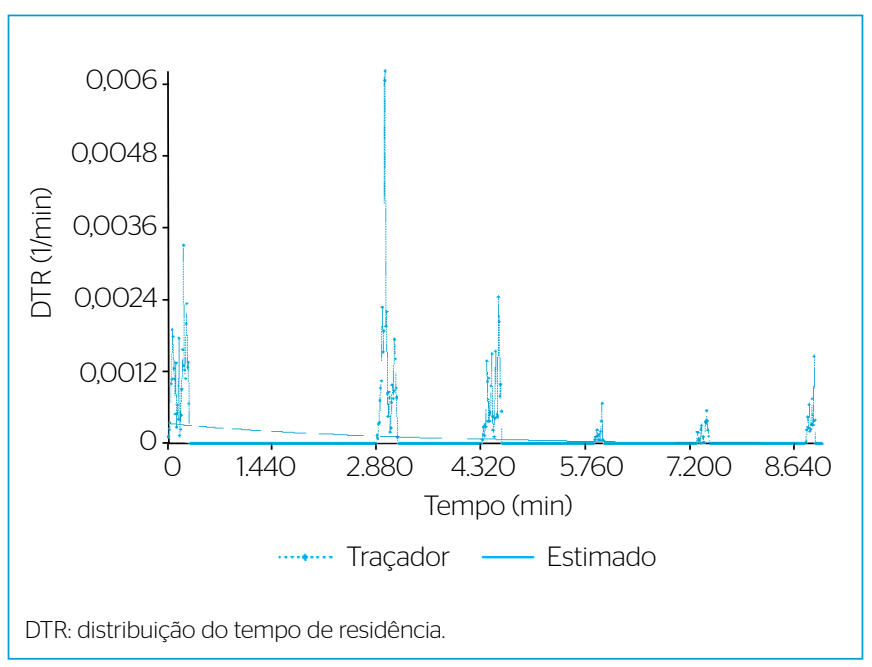

Figura 6 - Curva e ajuste da distribuição do tempo de residência pela função gama no $4^{\circ}$ teste, totalizando 150,5 horas (9.030 minutos).
No $2^{\circ}$ teste, a curva resposta de DTR apresentou características assimétricas da distribuição e mostrou a presença do prolongamento em forma de uma cauda longa, representando a existência de zonas com recirculação interna e zonas mortas (CASTRO, 2010; LEVENSPIEL, 2000). Essas zonas são provenientes de cantos, arestas ou espaços abaixo dos difusores de ar. Nota-se que, nas primeiras horas do experimento, uma quantidade de potássio foi conduzida rapidamente para fora da unidade, levando ao adiantamento do pico máximo do traçador. O restante decaiu lentamente no decorrer do período de avaliação, induzindo atraso na saída. Verifica-se que esses vários fatores vão ao encontro do modelo de dispersão de grande intensidade e com forte tendência ao escoamento com elevada mistura. Comportamento semelhante foi observado por Capela et al. (2009) e Lourenço \& Campos (2009), em que, respectivamente, o aparecimento de uma longa cauda mostrou familiaridade com os modelos em mistura completa e em dispersão de grande intensidade. $\mathrm{O}$ valor de $\mathrm{N}$ demonstra que a CG se mostrou bem misturada pelo sistema de aeração e vai ao

Tabela 5 - Resultados dos parâmetros hidrodinâmicos no $2^{\circ}$ e $4^{\circ}$ testes, com a caixa de gordura funcionando por flotação forçada.

\begin{tabular}{l|c|c|c} 
Parâmetro & Equação & $2^{\circ}$ teste & $4^{\circ}$ teste \\
\hline $\mathrm{TRH}_{\text {teórico }}$ & 1 & $1.232 \mathrm{~min}=0,86$ dias & $462 \mathrm{~min}=0,32$ dias \\
\hline $\mathrm{TRH}_{\text {médio }}$ & 2 & $700 \mathrm{~min}=0,49$ dias & $2.957 \mathrm{~min}=2,05$ dias \\
\hline$\sigma^{2}$ & 3 & $888.929,0 \mathrm{~min}^{2}$ & $5.780 .416,58 \mathrm{~min}^{2}$ \\
\hline$\sigma^{2} \theta$ & 4 & 1,82 & 0,66 \\
\hline$d$ & 5 & $*$ & 0,72 \\
\hline$N$ & 6,7 e 8 & 1 & $2 \mathrm{e} 1$ \\
\hline
\end{tabular}

$\mathrm{TRH}_{\text {médio: }}$ tempo de residência hidráulica médio; $\mathrm{TRH}_{\text {teórico: }}$ tempo de residência hidráulica teórico.

Não foi possível determinar o valor de $d$ pelo modelo de dispersão de grande intensidade utilizando "solver" na resolução da Equação 8.

Tabela 6 - Resultados dos índices encontrados na avaliação do desempenho hidráulico da caixa de gordura funcionando por flotação forçada no $2^{\circ}$ e $4^{\circ}$ testes hidrodinâmicos.

\begin{tabular}{|c|c|c|c|}
\hline Índices & 20 teste & $4^{\circ}$ teste & Interpretação (METCALF \& EDDY, 2003) \\
\hline Índice de avaliação da eficiência volumétrica $\left(\mathrm{TDH}_{\text {real }} / \mathrm{TDH}_{\text {teórico }}\right)$ & 0,57 & 6,39 & $\begin{array}{l}\text { Valor diferente de } 1 \text { indica perturbações de escoamento (zonas } \\
\text { mortas e/ou curtos-circuitos). }\end{array}$ \\
\hline Índice de tempo modal de residência $\left(\mathrm{TDH}_{\text {pico }} / \mathrm{TDH}_{\text {teórico }}\right)$ & 0,01 & 1,01 & $\begin{array}{l}\text { Valores próximos a zero indicam escoamento em mistura completa } \\
\text { e distribuição não uniforme com prováveis curtos-circuitos. Valor } \\
\text { próximo a } 1 \text { indica tendência a escoamento pistonado. }\end{array}$ \\
\hline Índice de curto-circuito $\left(\mathrm{TDH}_{\mathrm{i}} / \mathrm{TDH}_{\text {teórico }}\right)$ & 0,00 & 0,00 & $\begin{array}{l}\text { Valor igual a zero indica escoamento em mistura completa e } \\
\text { valor menor que } 1 \text { indica presença de curtos-circuitos. }\end{array}$ \\
\hline Índice de tempo de residência médio $\left(\mathrm{TDH}_{50 \%} / \mathrm{TDH}_{\text {real }}\right)$ & 0,32 & 1,02 & $\begin{array}{l}\text { Valor menor que } 1 \text { indica áreas estagnadas. Valor próximo a } 1 \\
\text { indica que as áreas estagnadas são insignificantes. }\end{array}$ \\
\hline Índice de dispersão de Morril (IDM=TDH $\left.{ }_{90 \%} / \mathrm{TDH}_{10 \%}\right)$ & 49,86 & 47,88 & $\begin{array}{l}\text { Valor>22 indica tendência a escoamento em mistura completa } \\
(\mid \mathrm{DM}>22) \text { com dispersão, devido à turbulência do ar. }\end{array}$ \\
\hline
\end{tabular}

$\mathrm{TRH}_{\text {médio }}$ : tempo de residência hidráulica médio; $\mathrm{TRH}_{\text {teóricio }}$ : tempo de residência hidráulica teórico; $\mathrm{TRH}_{\text {pico }}$ : tempo em que a concentração de pico do traçador é registrada na saída; TRH: tempo em que o traçador foi detectado pela primeira vez; $\mathrm{TRH}_{10 \%}, \mathrm{TRH}_{50 \%}$ e $\mathrm{TRH}_{90 \%}$ : tempo em que, respectivamente, 10,50 e $90 \%$ da massa do traçador passam pela saída da unidade; IDM: índice de dispersão de Morril. 
encontro do comportamento hidrodinâmico de mistura completa ideal (uma unidade). Os curtos-circuitos foram confirmados pelo valor de $\mathrm{TRH}_{\text {pico }} / \mathrm{TRH}_{\text {teórico }}$.

No $4^{\circ}$ teste, assim como no $3^{\circ}$, a curva de DTR não apresentou bom ajuste. $\mathrm{O}$ valor de $d$ aponta para um escoamento tendendo à condição de mistura completa com dispersão de grande intensidade (d>0,25) (METCALF \& EDDY, 2003). A dispersão observada resulta da turbulência do ar, injetado pelo soprador por meio dos difusores no fundo da CG, com a intercalada condição estacionária ao cessar a vazão. O traçador foi novamente agitado na CG quando voltava à vazão. Também a agitação ocorria com a vazão de pico e a inversão térmica. $\mathrm{O}$ valor de $N$ confirma a tendência ao escoamento em mistura completa. Comparativamente, Silva (2004) encontrou $d=0,49$ e $N=4$ para o sistema com aeração difusa (tanque de aeração), o que permitiu a caracterização de escoamento dispersivo próximo à mistura completa. Ainda nesse teste, $\mathrm{o}$ $\mathrm{TRH}_{\text {médio }}$ encontrado também superou o $\mathrm{TRH}_{\text {teórico }}$, sugerindo atraso na liberação (resposta) do traçador, confirmando a existência de zonas mortas no interior da CG, possivelmente devido ao volume líquido posicionado abaixo dos aeradores. Cabe mencionar que entre os difusores de ar e o fundo da CG há um espaço com valor médio em torno de $14 \mathrm{~cm}$, favorecendo, definitivamente, a criação de zonas mortas.

\section{CONCLUSÕES}

A partir dos ensaios hidrodinâmicos, concluiu-se que, com a CG tratando água residuária do RU em condições de flotação gravitacional, o escoamento foi dispersivo moderado, tendendo a pistonado, mas possuindo intrinsecamente certa mistura. Os desvios de escoamento foram ocasionados pelas zonas mortas, pelos curtos-circuitos e pela recirculação interna, efeitos que contribuem para a distribuição não uniforme. A determinação do modelo de escoamento sofreu influência de vários fatores: biomassa (lodo); retenção bem mais elevada de particulados menos densos nas camadas superiores (escuma) e de O\&G em maior quantidade; e, ainda, retenção de sólidos grosseiros mais densos por decantação na parte inferior. Esses fatores retardaram o escoamento e, consequentemente, o tempo de percurso do fluido na referida unidade.

Em condições de flotação forçada, com o sistema de aeração em funcionamento, é fortemente confirmada a tendência de escoamento em mistura dispersiva de elevada intensidade. $\mathrm{O}$ aumento do tempo de residência hidráulica relacionou-se à recirculação das partículas de fluido dentro da própria unidade, bem como às zonas mortas causadas pelos cantos prismáticos, ao volume líquido abaixo dos sopradores e mesmo outras arestas intrínsecas às unidades hidráulicas e de condução de ar internas da CG.

Apesar de não fazer parte do escopo deste trabalho, é importante salientar a eficiência da CG na remoção de gordura, levando-se em consideração os processos de flotação forçada e gravitacional. A CG mostrou maior eficiência na remoção de gorduras devido à menor perturbação hídrica quando operada por flotação gravitacional, uma vez que o fluxo, neste caso, tende a ser pistonado com menor grau de dispersão. Quando o sistema foi operado por flotação forçada, observou-se elevado grau de turbulência e, como previsto, menor eficiência na remoção de gordura, uma vez que ficou demonstrado que esse tipo de mistura possui elevada dispersão, tendendo para regime completamente misturado. Vale dizer ainda que, operando com flotação forçada, a gordura é bastante oxidada, formando uma escuma clara e de pequena espessura na parte superior da CG, minimizando, assim, problemas posteriores nas subsequentes unidades de tratamento.

\section{REFERÊNCIAS}

CAMPOS, C.M.M. (1990) Physical aspects affecting granulation in UASB Reactors. 459p. Thesis (PhD) - University of Newcastle upon Tyne, Newcastle.

CAPELA, l.; BILÉ, M.J.; SILVA, F:; NADAIS, H.; PRATES, A.; ARROJA, L. (2OO9) Hydrodynamic behaviour of a full-scale anaerobic contact reactor using residence time distribution technique. Journal of Chemistry Technology and Biotechnology, Londres, v. 84, n. 5, p. 716-724.

CASTRO, F.M.S. (2010) Estudo do comportamento hidrodinâmico de uma nova proposta de configuração interna para reator UASB. 102p. Dissertação (Mestrado em Recursos Hídricos e Saneamento Ambiental) - Universidade Federal do Rio Grande do Sul, Porto Alegre.
FERREIRA, D.C. (2012) Pós-tratamento de áqua residuária da suinocultura em sistemas alagados construídos combinado. 227 p. Tese (Doutorado em Saneamento Rural) - Universidade Federal de Lavras, Lavras.

JORDÃO, E.P. \& PESSÔA, C.A. (2005) Tratamento de esgotos domésticos. 3. ed. Rio de Janeiro: ABES.

KADLEC, R.H. \& WALLACE, R.D. (2008) Treatment wetlands. 2. ed. Flórida: CRC. 1016p.

LEVENSPIEL, O. (2000) Engenharia das reações químicas. 3. ed São Paulo: Blücher. v. 2. 563p. 
LOURENÇO, A.M.P. \& CAMPOS, C.M.M. (2009) Hydrodynamic behavior of lab-Scale Upflow Anaerobic Sludge Blanket (UASB) operated with an adopted hydraulic retention time (HRT) of 12 Hours. Ciência e Agrotecnologia, Lavras, v. 33, n. 4, p. 1139-1144.

MENDONÇA, L.C. (2002) Microbiologia e cinética de sistema de lodos ativados como pós-tratamento de efluente de reator anaeróbio de leito expandido. 240 p. Tese (Doutorado em Engenharia Civil) - Escola de Engenharia, Universidade de São Paulo, São Carlos.

METCALF \& EDDY. (2003) Wastewater engineering: treatment, disposal, reuse. 4. ed. New York: McGraw Hill. 1848p.

ORSSATO, F.; HERMES, E.; VILAS BOAS, M.A. (2010) Eficiência da remoção de óleos e graxas de uma estação de tratamento de esgoto sanitário, Cascavel. Engenharia Ambiental, Espírito Santo do Pinhal, v. 7, n. 4, p. 249-256.

PALMEIRA, V.A.A.; SILVA, S.R.; SOLETTI, J.I; CARVALHO, S.H.V. (2009) Tratamento de efluentes da indústria do coco utilizando os processos de coagulação e flotação por ar induzido. In: Congresso Brasileiro de Engenharia Química em Iniciação Científica, 8., 2009, Uberlândia. Anais... Uberlândia: UFU.

PAOLI, A.C. \& VON SPERLING, M. (2013) Avaliação das condições hidrodinâmicas de wetlands de escoamento horizontal subsuperficial (unidades plantada e não plantada). Revista Eletrônica de Gestão e Tecnologias Ambientais, v. 1, n. 2, p. 213-222.

SILVA, M.C.L. (2004) Avaliação de processos biológicos na melhoria da qualidade hídrica do canal Derby-Tacaruna. 100 p. Dissertação (Mestrado em Engenharia Química) - Universidade Federal de Pernambuco, Recife.

VON SPERLING, M. (1996) Princípios básicos do tratamento de esgotos. 5. ed. Belo Horizonte: UFMG. 211p. (Princípios do Tratamento Biológico de Águas Residuárias, 2).

YANG, B.; CHEN, G.; CHEN, G. (2012) Submerged membrane bioreactor in treatment of simulated restaurant wastewater. Separation and Purification Technology, Amsterdam, v. 88, p. 184-190. 